\title{
Exact Solution of the Marchenko Equation Relevant to Inverse Scattering on the Line
}

\section{Cornelis van der Mee*}

This paper presents explicit solutions of the Marchenko equation relevant to the solution of the inverse scattering problem of determining the real potential $Q(x)$ in the 1-D Schrödinger equation on the line from the reflection coefficient $R(k)$, which is assumed to be rational. The reflection coefficient is written in the form $i \mathcal{C}(k-i \mathcal{A})^{-1} \mathcal{B}$. State space methods are applied to solve the Marchenko equation, both without and with bound states.

\section{Introduction}

Consider the Schrödinger equation

$$
\psi^{\prime \prime}(k, x)+k^{2} \psi(k, x)=Q(x) \psi(k, x), \quad x \in \mathbf{R},
$$

where $\mathbf{R}$ is the real line, the prime denotes the derivative with respect to the spatial coordinate $x, k$ is the wavenumber, $k^{2}$ is energy, and $Q(x)$ is a real potential that is at least integrable on the line. Then the Jost solution from the left $f_{l}(k, x)$ and the Jost solution from the right $f_{r}(k, x)$ are the solutions of $(1.1)$ satisfying the boundary conditions

$$
f_{l}(k, x)= \begin{cases}e^{i k x}+o(1), & x \longrightarrow+\infty, \\ \frac{1}{T(k)} e^{i k x}+\frac{L(k)}{T(k)} e^{-i k x}+o(1), & x \rightarrow-\infty,\end{cases}
$$

$$
f_{r}(k, x)= \begin{cases}\frac{1}{T(k)} e^{-i k x}+\frac{R(k)}{T(k)} e^{i k x}+o(1), & x \rightarrow+\infty, \\ e^{-i k x}+o(1), & x \rightarrow-\infty,\end{cases}
$$

where $0 \neq k \in \mathbf{R}, T(k)$ is the transmission coefficient and $R(k)$ and $L(k)$ are the reflection coefficients from the right and from the left, respectively. The scattering matrix $\mathbf{S}(k)$ is given by

$$
\mathbf{S}(k)=\left[\begin{array}{cc}
T(k) & R(k) \\
L(k) & T(k)
\end{array}\right], \quad k \in \mathbf{R} .
$$

*This material is based on work supported by C.N.R., MURST, and a University of Cagliari Coordinated Research Project. 
Apart from the scattering solutions, one should also consider the nontrivial solutions of (1.1) that are square integrable on the real line, its so-called boundstate solutions. Such solutions occur at the values of $k$ in the open upper half-plane $\mathrm{C}^{+}$where the Jost solutions from the left and from the right are linearly dependent. When $(1+|x|) Q(x)$ is integrable, there are at most finitely many such $k$, all of which are purely imaginary. Moreover, for such potentials the reflection and transmission coefficients are continuous in $k \in \mathbf{R}$. For later use, let $L_{q}^{1}(I)$ denote the set of measurable functions $f$ on the interval $I$ such that $\|f\|_{1, q}:=\int_{I} d x(1+|x|)^{q}|f(x)|$ is finite.

The direct and inverse scattering theory for the Schrödinger equation (1.1) has been studied intensively $[13,15-18]$. The inverse problem that one usually considers, consists of the determination of a real potential $Q \in L_{1}^{1}(\mathbf{R})$ from the reflection coefficient $R(k)$, the bound state poles $i \kappa_{j}$ of the transmission coefficient $T(k)$, and the bound state constants $c_{j}=f_{r}\left(i \kappa_{j}, x\right) / f_{l}\left(i \kappa_{j}, x\right)(j=1, \ldots, \mathcal{N} ; \mathcal{N}$ finite, where there might not be any bound states). Necessary and sufficient conditions to construct $Q(x)$ from these data have been given in [15] for $Q \in L_{2}^{1}(\mathbf{R})$ and in [29] for $Q \in L_{1}^{1}(\mathbf{R})$. For quite general $R(k)$, an inversion algorithm based on the Marchenko integral equation was given by Faddeev [18] (see also [15, 16], and [13], Chapter XVII). The unique solvability of the Marchenko integral equation was proved in [16, 18], also when bound state information is incorporated.

When using the so-called Faddeev functions

$$
m_{l}(k, x)=e^{-i k x} f_{l}(k, x), \quad m_{r}(k, x)=e^{i k x} f_{r}(k, x),
$$

one obtains the Riemann-Hilbert problem

$$
\left[\begin{array}{c}
m_{l}(-k, x) \\
m_{r}(-k, x)
\end{array}\right]=\left[\begin{array}{cc}
T(k) & -R(k) e^{2 i k x} \\
-L(k) e^{-2 i k x} & T(k)
\end{array}\right]\left[\begin{array}{c}
m_{r}(k, x) \\
m_{l}(k, x)
\end{array}\right], \quad k \in \mathbf{R} .
$$

Since there exist integrable functions $B_{l}(x, \cdot), B_{r}(x, \cdot), \hat{R}$ and $\hat{L}$ such that

$$
\begin{gathered}
m_{l}(k, x)=1+\int_{0}^{\infty} d y e^{i k y} B_{l}(x, y), \quad m_{r}(k, x)=1+\int_{0}^{\infty} d y e^{i k y} B_{r}(x, y) ; \\
R(k)=\int_{-\infty}^{\infty} d z e^{-i k z} \hat{R}(z), \quad L(k)=\int_{-\infty}^{\infty} d z e^{-i k z} \hat{L}(z),
\end{gathered}
$$

when $Q \in L_{1}^{1}(\mathbf{R})$ [15], one can, by Fourier transformation and some calculus of residues, convert the Riemann-Hilbert problem (1.3) into the Marchenko integral equations

$$
\begin{aligned}
& B_{l}(x, y)+\int_{0}^{\infty} d z \hat{S}_{l}(2 x+y+z) B_{l}(x, z) \\
& \quad=-\hat{S}_{l}(2 x+y), \quad y>0
\end{aligned}
$$




$$
\begin{gathered}
B_{r}(x, y)+\int_{0}^{\infty} d z \hat{S}_{r}(-2 x+y+z) B_{r}(x, z) \\
\quad=-\hat{S}_{r}(-2 x+y), \quad y>0 .
\end{gathered}
$$

Here $\hat{S}_{l}$ and $\hat{S}_{r}$ coincide with $\hat{R}$ and $\hat{L}$, respectively, when there are no bound states. Otherwise

$$
\hat{S}_{l}(z)=\hat{R}(z)-i \sum_{j=1}^{\mathcal{N}} t_{j} c_{j} e^{-\kappa_{j} z}, \quad \hat{S}_{r}(z)=\hat{L}(z)-i \sum_{j=1}^{\mathcal{N}} \frac{t_{j}}{c_{j}} e^{-\kappa_{j} z}
$$

where $i \kappa_{1}, \ldots, i \kappa_{\mathcal{N}}$ are the poles of the transmission coefficient $T(k), t_{1}, \ldots, t_{\mathcal{N}}$ are the residues of $T(k)$ at these (simple) poles, and $c_{j}=f_{r}\left(i \kappa_{j}, x\right) / f_{l}\left(i \kappa_{j}, x\right)$ $(j=1, \ldots, \mathcal{N})$ are the bound state constants. It can be shown that $-i t_{j} c_{j}>0$ $(j=1, \ldots, \mathcal{N})$ [see Section 3; also [16]). The potential $Q(x)$ is easily found from the solution of either (1.4a) or (1.4b), since we have

$$
B_{l}\left(x, 0^{+}\right)=\frac{1}{2} \int_{x}^{\infty} d t Q(t), \quad B_{r}\left(x, 0^{+}\right)=\frac{1}{2} \int_{-\infty}^{x} d t Q(t) .
$$

Jump discontinuities in either of $B_{l}\left(x, 0^{+}\right)$and $B_{r}\left(x, 0^{+}\right)$lead to Dirac delta function terms in $Q(x)$ and hence to an extension of the class of potentials considered. Derivations of (1.4a) and (1.4b) can be found in [13,16].

The inverse scattering problem for rational reflection coefficients is easily solved in an ad hoc way by computing $B_{l}(x, y)$ for $x \geq 0\left[B_{r}(x, y)\right.$ for $x \leq 0$, respectively] from (1.3) using calculus of residues, where the values of $m_{l}(k, x)$ at the poles of $R(k)$ in $\mathbf{C}^{+}$when $x \geq 0$ [the values of $m_{r}(k, x)$ at the poles of $L(k)$ in $\mathbf{C}^{+}$ when $x \leq 0$, respectively] follow by solving a linear system of equations (cf. [2] and references therein). However, in this article we apply state space methods (see, e.g., [9]) which allow us to derive explicit formulas for thet solution of the above inverse scattering problem when $R(k)$ is a rational function without real poles that is real-valued for purely imaginary $k$, vanishes as $k \rightarrow \infty$, and satisfies $|R(k)|<1$ for $0 \neq k \in \mathbf{R}$ and $R(0) \in[-1,1)$. We then have the state space realization

$$
R(k)=i \mathcal{C}(k-i \mathcal{A})^{-1} \mathcal{B}=i\left((k-i \mathcal{A})^{-1} \beta, \gamma\right) \text {, }
$$

where $\mathcal{A}$ is a real $n \times n$ matrix without imaginary eigenvalues, $\mathcal{B}$ is a real $n \times 1$ matrix coinciding with the column vector $\beta, \mathcal{C}$ is a real $1 \times n$ matrix coinciding with the row vector $\gamma^{T}$, and $(\cdot, \cdot)$ denotes the usual scalar product on $\mathbf{C}^{n}$. Writing

$$
(k-i \mathcal{A})^{-1}=i \int_{-\infty}^{\infty} d t e^{-i k t} E(t ;-\mathcal{A}),
$$

the solution $Q(x)$ of the inverse problem follows from the solution $B_{l}(x, y)$ of the Marchenko integral equation

$$
\begin{aligned}
& B_{l}(x, y)-\int_{0}^{\infty} d z \mathcal{C} E(2 x+y+z ;-\mathcal{A}) \mathcal{B} B_{l}(x, z) \\
& \quad=\mathcal{C} E(2 x+y ;-\mathcal{A}) \mathcal{B}, \quad y>0
\end{aligned}
$$


by means of the formula [cf. (1.5)]

$$
Q(x)=-2 \frac{d}{d x} B_{!}\left(x, 0^{+}\right),
$$

when there are no bound states. In fact, we shall prove the following theorem.

Theorem 1.1 Suppose there are no bound states. Then for $x>0$ the unique solution of (1.8) is given by

$$
B_{l}(x, y)=\mathcal{C} E(2 x+y ;-\mathcal{A}) \Lambda(x)^{-1} \mathcal{B},
$$

and the potential $Q(x)$ is given by

$$
\begin{aligned}
Q(x) & =4 \mathcal{C} E(2 x ;-\mathcal{A}) \Lambda(x)^{-1} \mathcal{A} \Lambda(x)^{-1} \mathcal{B} \\
& =4\left(E(2 x ;-\mathcal{A}) \Lambda(x)^{-1} \mathcal{A} \Lambda(x)^{-1} \beta, \gamma\right),
\end{aligned}
$$

where

$$
\Lambda(x)=I-\mathcal{D} E(2 x ;-\mathcal{A})
$$

and

$$
\mathcal{D}=\int_{0}^{\infty} d u E(u ;-\mathcal{A}) \mathcal{B C} E(u ;-\mathcal{A}) .
$$

When there are bound states, i.e., when $T(k)$ has finitely many (simple) poles at $i \kappa_{j}$ with residue $t_{j}$ and bound state constant $c_{j}$ with $d_{j}=-i t_{j} c_{j}>0(j=$ $1, \ldots, \mathcal{N})$, the Marchenko integral equation is given by $(1.4 \mathrm{a})$, where

$$
\hat{S}_{l}(z)=-\mathcal{C} E(z ;-\mathcal{A}) \mathcal{B}+\sum_{j=1}^{\mathcal{N}} d_{j} e^{-\kappa_{j} z}=-\breve{C} E(z ;-\breve{A}) \breve{B},
$$

and $\breve{A}, \breve{B}$ and $\check{C}$ are the real $(n+\mathcal{N}) \times(n+\mathcal{N}),(n+\mathcal{N}) \times 1$ and $1 \times(n+\mathcal{N})$ matrices given by

$$
\begin{aligned}
\check{\mathcal{A}} & =\mathcal{A} \oplus \operatorname{diag}\left(\kappa_{1}, \ldots, \kappa_{\mathcal{N}}\right), \quad \check{\mathrm{B}}=\left[\mathcal{B}-\mathrm{d}_{1} \ldots-\mathrm{d}_{\mathcal{N}}\right]^{\mathrm{T}}, \\
\check{\mathcal{C}} & =\left[\begin{array}{lll}
\mathcal{C} & 1
\end{array} \ldots 1\right] .
\end{aligned}
$$

We also write $\breve{\beta}=\left[\begin{array}{llll}\beta-d_{1} \ldots & \ldots-d_{\mathcal{N}}\end{array}\right]^{T}$ and $\check{\gamma}=\left[\begin{array}{llll}\gamma & 1 & \ldots & 1\end{array}\right]^{T}$. We then have the following theorem.

Theorem 1.2 For $x>0$ the unique solution of the Marchenko equation (1.4a) is given by

$$
B_{l}(x, y)=\breve{\mathcal{C}} E(2 x+y ;-\breve{\mathcal{A}}) \breve{\Lambda}(x)^{-1} \breve{\mathcal{B}},
$$


and the potential $Q(x)$ is given by

$$
\begin{aligned}
Q(x) & =4 \breve{\mathcal{C}} E(2 x ;-\breve{\mathcal{A}}) \breve{\Lambda}(x)^{-1} \breve{\mathcal{A}} \breve{\Lambda}(x)^{-1} \breve{\mathcal{B}} \\
& =4\left(E(2 x ;-\breve{\mathcal{A}}) \breve{\Lambda}(x)^{-1} \breve{\mathcal{A}} \breve{\Lambda}(x)^{-1} \breve{\beta}, \breve{\gamma}\right),
\end{aligned}
$$

where

$$
\breve{\Lambda}(x)=I-\breve{\mathcal{D}} E(2 x ;-\breve{\mathcal{A}}), \quad \breve{\mathcal{D}}=\int_{0}^{\infty} d u E(u ;-\breve{\mathcal{A}}) \breve{\mathcal{B}} \breve{C} E(u ;-\breve{\mathcal{A}}) .
$$

Since $E(t ;-\mathcal{A})$ is a so-called bisemigroup $[10,11]-$ we shall give its definition below,$-(1.8)$ is an integral equation with a separable kernel when $x>0$, which makes it trivial to solve. When $x<0$, there are two approaches. The first is to solve (1.4b) for $x<0$, where $\hat{S}_{r}(z)$ is written as in (1.14) and which is an integral equation with separable kernel. The other approach is to continue studying (1.8) which leads to

$$
B_{l}(x, y)=\mathcal{C} E(2 x+y ;-\mathcal{A}) \omega(x), \quad y>-2 x,
$$

for a suitable vector $\omega(x)$. Then for $0<y<-2 x,(1.8)$ is first written as a system of integral equations for the vector with components $B_{l}(x, y)$ and $\tilde{B}_{l}(x, y)=$ $B_{l}(x,-2 x-y)$. Since this system has a so-called semi-separable kernel, it could in principle be solved using the methods of [21], Chapter IX. Instead, we will reduce this coupled set of integral equations to a linear system governed by a first order linear differential equation and solve it by elementary means, yielding $Q(x)$ for $x<0$ as the final result. This program will first be carried out if there are no bound states (Section 2) and then when bound states are taken into account (Section 3). The potential $Q(x)$ will contain the additional term $\left(\lim _{k \rightarrow \infty} 2 i k R(k)\right) \delta(x)=$ $-2 \mathcal{C B} \delta(x)$ where $\delta(x)$ is the Dirac delta function [see (1.6), (1.8), and (1.9)]; this term vanishes if $R(k)=o(1 / k)$ as $k \rightarrow \infty$. Finally, in the Appendix we give a concise proof of the unique solvability of the Marchenko integral equations.

The inverse problem for the Schrödinger equation (1.1) on the half-line $x \in$ $(0,+\infty)$ has basically been solved in the 1950's [1, 17, 19, 20, 27, 28]. This problem consists of the determination of a real potential $Q(x)$ from the spectral function of the differential operator $-\left(d^{2} / d x^{2}\right)+Q(x)$ with Dirichlet boundary condition at $x=0$. In recent years state space methods have been used to derive the exact solution of this problem with rational spectral function and of the problem of computing the potential from a rational scattering function. This was done for the usual Schrödinger equation [24] and for the so-called canonical differential operators [3-7, 22, 23].

When the research leading to the present article was completed, the author learned of the existence of [8], where the state space method is applied to the inverse scattering problem for the $n \times n$ matrix Schrödinger equation on the full line with selfadjoint potential $Q(x)$ and rational reflection coefficient $R(k)=o(1 / k)$ as $k \rightarrow \infty$. In the present paper a different method is used to arrive at explicit 
solutions of the inverse problem. Our paper is not based on [34] (which is in turn based on [35]), where the inverse scattering theory of the $n \times n$ matrix Schrödinger equation has not been fully developed. The articles $[8,34,35]$ will be discussed in more detail in a later publication.

\section{Solution of the Inverse Problem without Bound States}

In this paper we distinguish between the generic and the exceptional case [15, 18]. Generically $f_{l}(0, x)$ and $f_{r}(0, x)$ are linearly' independent and $T(k) \sim i c k, k \rightarrow 0$ in $\overline{\mathbf{C}^{+}}$, where $0 \neq c \in \mathbf{R}$. We then have

$$
T(0)=0, \quad R(0)=L(0)=-1 .
$$

In the so-called exceptional case these two functions are linearly dependent and $T(k)=T(0)+o(1), k \rightarrow 0$ in $\overline{\mathbf{C}^{+}}$, for some $0 \neq T(0) \in \mathbf{R}([15,18]$ if $Q \in L_{2}^{1}(\mathbf{R})$; [26] if $Q \in L_{\mathrm{i}}^{1}(\mathbf{R})$ ). Thus the modulus of $T(k)$ is known; the final form of $T(k)$ now depends on the presence of bound states or not.

\section{A. Basic Concepts}

Let $\mathcal{A}$ be an arbitrary real $n \times n$ matrix without zero or imaginary eigenvalues and let $\sigma(\mathcal{A})$ denote its set of eigenvalues. Let $\Gamma^{(+)}$and $\Gamma^{(-)}$be positively oriented simple Jordan contours in the right and left half-plane enclosing all of the eigenvalues of $\mathcal{A}$ in the open right and left half-plane, respectively. Let $P_{A}^{(+)}$and $P_{\mathcal{A}}^{(-)}$be the spectral projections of $\mathcal{A}$ corresponding to its eigenvalues in the right and left half-plane, respectively. For $0 \neq t \in \mathbf{R}$ we then define the bisemigroup generated by $\mathcal{A}$ by

$$
E(t ;-\mathcal{A})= \begin{cases}e^{-t \mathcal{A}} P_{\mathcal{A}}^{(+)}=\frac{1}{2 \pi i} \int_{\Gamma^{(+)}} e^{-t z}(z-\mathcal{A})^{-1} d z, & t>0 \\ -e^{-t \mathcal{A}} P_{\mathcal{A}}^{(-)}=-\frac{1}{2 \pi i} \int_{\Gamma^{(-)}} e^{-t z}(z-\mathcal{A})^{-1} d z, & t<0 .\end{cases}
$$

Then (1.7) holds true. For later use, let $\mathcal{J}_{\mathcal{A}}^{( \pm)}$be the natural imbedding of the range of $P_{\mathcal{A}}^{( \pm)}$into $\mathbf{C}^{n}$ and $\pi_{\mathcal{A}}^{( \pm)}$the unique operator from $\mathbf{C}^{n}$ onto the range of $P_{\mathcal{A}}^{( \pm)}$such that $\mathcal{J}_{\mathcal{A}}^{( \pm)} \pi_{\mathcal{A}}^{( \pm)}=P_{\mathcal{A}}^{( \pm)}$.

\section{B. Reflection and Transmission Coefficients}

Let $R(k)$ be a rational function of $k$ without real poles vanishing at infinity and satisfying $R(-k)=\overline{R(k)}$ for $k \in \mathbf{R}$. Then there exist real matrices $\mathcal{A}$ (of size $n \times n$ ), $\mathcal{B}$ (of size $n \times 1$ and thus representable as the column vector $\beta$ ) and $\mathcal{C}$ (of 
size $1 \times n$ and thus representable as the row vector $\gamma^{T}$ ) such that (1.6) holds. In that case

$$
\hat{R}(t)=\frac{1}{2 \pi} \int_{-\infty}^{\infty} d k e^{i k t} R(k)=-\mathcal{C} E(t ;-\mathcal{A}) \mathcal{B}=-(E(t ;-\mathcal{A}) \beta, \gamma) .
$$

We require $n$ to be minimal. We put $\mathcal{C}_{ \pm}=\mathcal{C} \mathcal{J}_{\mathcal{A}}^{( \pm)}, \mathcal{A}_{ \pm}=\pi_{\mathcal{A}}^{( \pm)} \mathcal{A} \mathcal{J}_{\mathcal{A}}^{( \pm)}$and $\mathcal{B}_{ \pm}=\pi_{\mathcal{A}}^{( \pm)} \mathcal{B}$

Since the scattering matrix $\mathbf{S}(k)$ is unitary for $k \in \mathbf{R}$, we now easily compute

$$
|T(k)|^{2}=T(k) T(-k)=1-i\left[\begin{array}{ll}
\mathcal{C} & 0
\end{array}\right]\left[\begin{array}{cc}
k-i \mathcal{A} & i \mathcal{B C} \\
0 & k+i \mathcal{A}
\end{array}\right]^{-1}\left[\begin{array}{l}
0 \\
\mathcal{B}
\end{array}\right] .
$$

Moreover,

$$
\frac{1}{|T(k)|^{2}}=\frac{1}{T(k) T(-k)}=1+i\left[\begin{array}{ll}
\mathcal{C} & 0
\end{array}\right](k-i \mathcal{E})^{-1}\left[\begin{array}{l}
0 \\
\mathcal{B}
\end{array}\right],
$$

where

$$
\mathcal{E}=\left[\begin{array}{cc}
\mathcal{A} & -\mathcal{B C} \\
\mathcal{B C} & -\mathcal{A}
\end{array}\right]
$$

Then $M \mathcal{E} M=-\mathcal{E}$ for $M=\left[\begin{array}{ll}0 & I \\ I & 0\end{array}\right]$, so that the spectrum of $\mathcal{E}$ is symmetric with respect to the origin.

Since $R(-k)=\overline{R(k)}$ for $k \in \mathbf{R}$ and the order of the matrix $\mathcal{A}$ in the realization (1.6) is minimal, there exists a unique nonsingular $J$ such that $J \mathcal{A}=\mathcal{A}^{*} J, J \mathcal{B}=$ $\mathcal{C}^{*}$ and $\mathcal{C}=\mathcal{B}^{*} J$, where the asterisk denotes the conjugate transpose. Then $J$ is selfadjoint, while

$$
(J \oplus(-J)) \mathcal{E}=\mathcal{E}^{*}(J \oplus(-J))
$$

implies that the spectrum $\sigma(\mathcal{E})$ of $\mathcal{E}$ is symmetric with respect to the real line. In the generic case $\mathcal{E}$ is nonsingular, whereas in the exceptional case $\mathcal{E}$ has a double zero eigenvalue.

The following well-known result $[12,32,33]$ is needed to compute integrals of the form (1.13) above. We will omit the proof.

Proposition 2.1 Let $\Gamma^{(+)}$and $\Gamma^{(-)}$be simple positively oriented Jordan contours enclosing all of the eigenvalues of $\mathcal{A}$ and $-\mathcal{A}$ in the right and left half-plane, respectively, and let

$$
\begin{aligned}
\mathcal{D} & =\frac{1}{2 \pi i} \int_{\Gamma^{(+)}} d z(z-\mathcal{A})^{-1} \mathcal{B C}(z+\mathcal{A})^{-1} \\
& =\frac{-1}{2 \pi i} \int_{\Gamma^{(-)}} d z(z-\mathcal{A})^{-1} \mathcal{B C}(z+\mathcal{A})^{-1} .
\end{aligned}
$$


Then $\mathcal{D}$ maps the range of $P_{\mathcal{A}}^{(+)}$into the range of $P_{\mathcal{A}}^{(+)}, \mathcal{D}^{\bullet}=\pi_{\mathcal{A}}^{(+)} \mathcal{D} \mathcal{J}_{\mathcal{A}}^{(+)}$is the unique solution of the matrix equation

$$
\mathcal{A}_{+} \mathcal{D}^{\bullet}+\mathcal{D}^{\bullet} \mathcal{A}_{+}=\pi_{\mathcal{A}}^{(+)} \mathcal{B C J}_{\mathcal{A}}^{(+)}
$$

where $\mathcal{A}_{+}=\pi_{\mathcal{A}}^{(+)} \mathcal{A} \mathcal{J}_{\mathcal{A}}^{(+)}$, and $\mathcal{D}$ is also given by (1.13).

C. Solution of the Marchenko Equation for $x>0$

Reducing (1.8) with $x>0$ to an integral equation for $C_{l}(x)=\int_{0}^{\infty} d z E(z ;-\mathcal{A})$ $\mathcal{B} B_{l}(x, z)$, we find that the solution of (1.8) for $x>0$ is given by

$$
B_{l}(x, y)=\mathcal{C} E(2 x+y ;-\mathcal{A}) \Lambda(x)^{-1} \mathcal{B},
$$

where $\Lambda(x)$ given by (1.12) is nonsingular. Indeed, assuming $\Lambda(x)$ singular would lead to a solution of the homogeneous counterpart to (1.8), which contradicts Theorem A.1. Using (1.5) and $\frac{d}{d x} \Lambda(x)^{-1}=:-\Lambda(x)^{-1}\left(\frac{d}{d x} \Lambda(x)\right) \Lambda(x)^{-1}$, we get (1.11) for $x>0$.

D. Solution of the Marchenko Equation for $x<0$

To solve (1.8), put

$$
C_{l}(x, y)=E(2 x+y ;-\mathcal{A}) \mathcal{B}+\int_{0}^{\infty} d z E(2 x+y+z ;-\mathcal{A}) \mathcal{B} B_{l}(x, z) .
$$

Then

$$
B_{l}(x, y)=\mathcal{C} C_{l}(x, y)
$$

and

$$
\begin{aligned}
& C_{l}(x, y)-\int_{0}^{\infty} d z E(2 x+y+z ;-\mathcal{A}) \mathcal{B C} C_{l}(x, z) \\
& =E(2 x+y ;-\mathcal{A}) \mathcal{B}, \quad y>0 .
\end{aligned}
$$

For $y>-2 x$ we easily obtain $(\partial / \partial y) C_{l}(x, y)=-\mathcal{A} C_{l}(x, y)$, where the entries of $C_{l}(x, y)$ are exponentially decreasing as $y \rightarrow+\infty$. Hence,

$$
C_{l}(x, y)=E(2 x+y ;-\mathcal{A}) \omega(x)
$$

for some vector $\omega(x)$ in the range of $P_{\mathcal{A}}^{(+)}$to be determined shortly.

Now consider (2.4) for $0<y<-2 x$. Putting $\tilde{C}_{l}(x, y)=C_{l}(x,-2 x-y)$ and using the identity [cf. (1.13)]

$$
\int_{-2 x}^{\infty} d z E(2 x+z ;-\mathcal{A}) \mathcal{B C} E(2 x+z ;-\mathcal{A}) \omega(x)=\mathcal{D} \omega(x)
$$


we obtain the coupled system of integral equations

$$
C_{l}(x, y)-\int_{0}^{-2 x} d z E(y-z ;-\mathcal{A}) \mathcal{B C} \tilde{C}_{l}(x, z)
$$

$$
\begin{gathered}
=E(2 x+y ;-\mathcal{A}) \mathcal{B}+E(y ;-\mathcal{A}) \mathcal{D} \omega(x) \\
\tilde{C}_{l}(x, y)-\int_{0}^{-2 x} d z E(z-y ;-\mathcal{A}) \mathcal{B C C} C_{l}(x, z) \\
=E(-y ;-\mathcal{A}) \mathcal{B}+E(-2 x-y ;-\mathcal{A}) \mathcal{D} \omega(x),
\end{gathered}
$$

where $0<y<-2 x$. As a result, we get

$$
\frac{\partial}{\partial y}\left[\begin{array}{c}
C_{l}(x, y) \\
\tilde{C}_{l}(x, y)
\end{array}\right]=-\mathcal{E}\left[\begin{array}{c}
C_{l}(x, y) \\
\tilde{C}_{l}(x, y)
\end{array}\right], \quad 0<y<-2 x,
$$

where $\mathcal{E}$ is given by (2.1). Putting $\mathcal{Q}_{\mathcal{A}}=P_{\mathcal{A}}^{(+)} \oplus P_{\mathcal{A}}^{(-)}$, one may write the boundary conditions in the concise form

$$
\begin{aligned}
\mathcal{Q}_{\mathcal{A}}\left[\begin{array}{c}
C_{l}\left(x, 0^{+}\right) \\
\tilde{C}_{l}\left(x, 0^{+}\right)
\end{array}\right] & =\left[\begin{array}{c}
\mathcal{D} \omega(x) \\
-P_{\mathcal{A}}^{(-)} \mathcal{B}
\end{array}\right], \\
\left(I-\mathcal{Q}_{\mathcal{A}}\right)\left[\begin{array}{c}
C_{l}\left(x,(-2 x)^{-}\right) \\
\tilde{C}_{l}\left(x,(-2 x)^{-}\right)
\end{array}\right] & =\left[\begin{array}{c}
-P_{\mathcal{A}}^{(-)} \mathcal{B} \\
\mathcal{D} \omega(x)
\end{array}\right],
\end{aligned}
$$

implying

$$
\left(\mathcal{Q}_{\mathcal{A}}+\left(I-\mathcal{Q}_{\mathcal{A}}\right) e^{2 x \mathcal{E}}\right)\left[\begin{array}{c}
C_{l}\left(x, 0^{+}\right) \\
\tilde{C}_{l}\left(x, 0^{+}\right)
\end{array}\right]=\left[\begin{array}{l}
I \\
I
\end{array}\right]\left(\mathcal{D} \omega(x)-P_{\mathcal{A}}^{(-)} \mathcal{B}\right)
$$

Now note that the matrix $\left(\mathcal{Q}_{\mathcal{A}}+\left(I-\mathcal{Q}_{\mathcal{A}}\right) e^{2 x \mathcal{E}}\right)$ is nonsingular; otherwise the homogeneous counterpart to the system (2.5) would have a nontrivial solution, which would contradict Corollary A.2. As a result,

$$
\left[\begin{array}{c}
C_{l}\left(x, 0^{+}\right) \\
\tilde{C}_{l}\left(x, 0^{+}\right)
\end{array}\right]=\left(\mathcal{Q}_{\mathcal{A}}+\left(I-\mathcal{Q}_{\mathcal{A}}\right) e^{2 x \mathcal{E}}\right)^{-1}\left[\begin{array}{l}
I \\
I
\end{array}\right]\left(\mathcal{D} \omega(x)-P_{\mathcal{A}}^{(-)} \mathcal{B}\right),
$$

which implies

$$
C_{l}(x, y)=\left[\begin{array}{ll}
I & 0
\end{array}\right] e^{-y \mathcal{E}}\left(\mathcal{Q}_{\mathcal{A}}+\left(I-\mathcal{Q}_{\mathcal{A}}\right) e^{2 x \mathcal{E}}\right)^{-1}\left[\begin{array}{l}
I \\
I
\end{array}\right]\left(\mathcal{D} \omega(x)-P_{\mathcal{A}}^{(-)} \mathcal{B}\right)
$$

It remains to determine $\omega(x)$. From (2.2) we derive the identity

$$
C_{l}\left(x,(-2 x)^{+}\right)-C_{l}\left(x,(-2 x)^{-}\right)=\mathcal{B}
$$


which implies [with the help of $P_{\mathcal{A}}^{(+)} \omega(x)=\omega(x)=C_{l}\left(x,(-2 x)^{+}\right)$]

$$
\begin{aligned}
\omega(x) & =\mathcal{B}+C_{l}\left(x,(-2 x)^{-}\right) \\
& =\mathcal{B}+\left[\begin{array}{ll}
I & 0
\end{array}\right]\left(\mathcal{Q}_{\mathcal{A}} e^{-2 x \mathcal{E}}+\left(I-\mathcal{Q}_{\mathcal{A}}\right)\right)^{-1}\left[\begin{array}{l}
I \\
I
\end{array}\right]\left(\mathcal{D} \omega(x)-P_{\mathcal{A}}^{(-)} \mathcal{B}\right),
\end{aligned}
$$

yielding

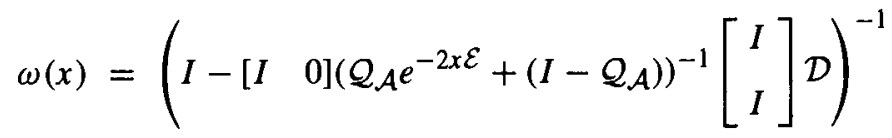

$$
\begin{aligned}
& \times\left(I-\left[\begin{array}{ll}
I & 0
\end{array}\right]\left(\mathcal{Q}_{\mathcal{A}} e^{-2 x \mathcal{E}}+\left(I-\mathcal{Q}_{\mathcal{A}}\right)\right)^{-1}\left[\begin{array}{l}
I \\
I
\end{array}\right] P_{\mathcal{A}}^{(-)}\right) \mathcal{B}
\end{aligned}
$$

where the inverse operator in the right-hand side exists. In fact, reasoning as above, we see that the unique solvability of (1.8) [see Theorem A.1] implies the nonsingularity of the two matrices $\mathcal{Q}_{\mathcal{A}}+\left(I-\mathcal{Q}_{\mathcal{A}}\right) e^{2 x \mathcal{E}}$ and $I-\left[\begin{array}{ll}I & 0\end{array}\right]\left(\mathcal{Q}_{\mathcal{A}} e^{-2 x \mathcal{E}}+\right.$ $\left.\left(I-\mathcal{Q}_{\mathcal{A}}\right)\right)^{-1}\left[\begin{array}{l}I \\ I\end{array}\right] \mathcal{D}$. Finally,

$$
B_{l}\left(x, 0^{+}\right)=\mathcal{C}\left[\begin{array}{ll}
I & 0
\end{array}\right]\left(\mathcal{Q}_{\mathcal{A}}+\left(I-\mathcal{Q}_{\mathcal{A}}\right) e^{2 x \mathcal{E}}\right)^{-1}\left[\begin{array}{l}
I \\
I
\end{array}\right]\left(\mathcal{D} \omega(x)-P_{\mathcal{A}}^{(-)} \mathcal{B}\right),
$$

yielding $Q(x)=-2 \frac{d}{d x} B_{l}\left(x, 0^{+}\right)$, which decreases exponentially as $x \rightarrow-\infty$. We omit the rather cumbersome expression for $Q(x)$ with $x<0$.

When $R(k)$ is analytic in $\mathbf{C}^{+}$and hence the matrix $\mathcal{A}$ in its realization (1.6) has only eigenvalues in the open left half-plane, we have $E(t ;-\mathcal{A})=-e^{-t \mathcal{A}}$ for $t<0$ and $E(t ;-\mathcal{A})=0$ for $t>0$. For $x<0$ the Marchenko integral equation (1.8) reduces to $B_{l}(x, y)=0$ for $y>-2 x$ and this leads to significant simplifications in the above inversion algorithm. Defining $C_{l}(x, y)$ as in (2.2), we get (2.3) and

$$
\begin{gathered}
C_{l}(x, y)+\int_{0}^{-2 x-y} d z e^{-(2 x+y+z) \mathcal{A}} \mathcal{B C} C_{l}(x, z) \\
=-e^{-(2 x+y) \mathcal{A}_{B}}, \quad 0<y<-2 x .
\end{gathered}
$$

Next we get the linear system of equations

$$
\begin{gathered}
C_{l}(x, y)+\int_{y}^{-2 x} d z e^{(z-y) \mathcal{A}} \mathcal{B C} \tilde{C}_{l}(x, z)=-e^{-(2 x+y) \mathcal{A}_{\mathcal{B}}} \\
\tilde{C}_{l}(x, y)+\int_{0}^{y} d z e^{(y-z) \mathcal{A}} \mathcal{B C}_{l}(x, z)=-e^{y \mathcal{A}} \mathcal{B}
\end{gathered}
$$


where $0<y<-2 x$ and $\tilde{C}_{l}(x, y)=C_{l}(x,-2 x-y)$. We then get the system of differential equations (2.6) where $\tilde{C}_{l}\left(x, 0^{+}\right)=C_{l}\left(x,(-2 x)^{-}\right)=-\mathcal{B}$. Hence

$$
\left[\begin{array}{c}
C_{l}(x, y) \\
\tilde{C}_{l}(x, y)
\end{array}\right]=e^{-y \mathcal{E}}\left[\begin{array}{l}
h_{1} \\
h_{2}
\end{array}\right],
$$

where

$$
\left[\begin{array}{cc}
{\left[e^{2 x \mathcal{E}}\right]_{11}} & {\left[e^{2 x \mathcal{E}}\right]_{12}} \\
0 & I
\end{array}\right]\left[\begin{array}{l}
h_{1} \\
h_{2}
\end{array}\right]=\left(\left[\begin{array}{ll}
0 & 0 \\
0 & I
\end{array}\right]+\left[\begin{array}{ll}
I & 0 \\
0 & 0
\end{array}\right] e^{2 x \mathcal{E}}\right)
$$

$$
\left[\begin{array}{l}
h_{1} \\
h_{2}
\end{array}\right]=\left[\begin{array}{c}
-\mathcal{B} \\
-\mathcal{B}
\end{array}\right] .
$$

Now note that the matrix $\left[e^{2 x \mathcal{E}}\right]_{11}$ is nonsingular; otherwise one could construct a nontrivial solution of the homogeneous counterpart of (2.8), which would contradict Corollary A.2. As a result,

$$
\left[\begin{array}{l}
h_{1} \\
h_{2}
\end{array}\right]=\left[\begin{array}{cc}
{\left[e^{2 x \mathcal{E}}\right]_{11}^{-1}} & -\left[e^{2 x \mathcal{E}}\right]_{11}^{-1}\left[e^{2 x \mathcal{E}}\right]_{12} \\
0 & I
\end{array}\right]\left[\begin{array}{l}
-\mathcal{B} \\
-\mathcal{B}
\end{array}\right] .
$$

Consequently,

$$
\begin{aligned}
B_{l}\left(x, 0^{+}\right) & =\mathcal{C C}_{l}\left(x, 0^{+}\right) \\
& =-\mathcal{C}\left[\begin{array}{ll}
I & 0
\end{array}\right]\left[\begin{array}{cc}
{\left[e^{2 x \mathcal{E}}\right]_{11}^{-1}} & -\left[e^{2 x \mathcal{E}}\right]_{11}^{-1}\left[e^{2 x \mathcal{E}}\right]_{12} \\
0 & I
\end{array}\right]\left[\begin{array}{l}
I \\
I
\end{array}\right] \mathcal{B} \\
& =-\left(\left[e^{2 x \mathcal{E}}\right]_{11}^{-1}\left(I-\left[e^{2 x \mathcal{E}}\right]_{12}\right) \beta, \gamma\right) .
\end{aligned}
$$

Using (1.5) we finally obtain for $x<0$

$$
Q(x)=-4\left(\left[e^{2 x \mathcal{E}}\right]_{11}^{-1}\left\{\left[\mathcal{E} e^{2 x \mathcal{E}}\right]_{11}\left[e^{2 x \mathcal{E}}\right]_{11}^{-1}\left(I-\left[e^{2 x \mathcal{E}}\right]_{12}\right)+\left[\mathcal{E} e^{2 x \mathcal{E}}\right]_{12}\right\} \beta, \gamma\right) .
$$

When $R(k)$ is analytic in $\mathbf{C}^{-}$and hence the matrix $\mathcal{A}$ in (1.6) has its eigenvalues in the open right half-plane, the situation is somewhat more complicated. For $x>0, Q(x)$ is given by (1.11). For $x<0$ we define $C_{l}(x, y)$ as in (2.2), and derive (2.3) and the integral equation

$$
C_{l}(x, y)-\int_{\max (0,-2 x-y)}^{\infty} d z e^{-(2 x+y+z) \mathcal{A}} \mathcal{B C} C_{l}(x, z)
$$

$$
= \begin{cases}e^{-(2 x+y) \mathcal{A}} \mathcal{B}, & y>-2 x \\ 0, & 0<y<-2 x .\end{cases}
$$


Since $C_{l}(x, y)=e^{-(2 x+y) \mathcal{A}} \omega(x)$ for $y>-2 x$, we obtain

$$
\begin{gathered}
C_{l}(x, y)-\int_{-2 x-y}^{-2 x} d z e^{-(2 x+y+z) \mathcal{A}} \mathcal{B C}_{l}(x, z) \\
=e^{-y \mathcal{A}} \mathcal{D} \omega(x), \quad 0<y<-2 x,
\end{gathered}
$$

where $\mathcal{D}$ is given by (1.13). Putting $\tilde{C}_{l}(x, y)=C_{l}(x,-2 x-y)$ we find

$$
\begin{gathered}
C_{l}(x, y)-\int_{0}^{y} d z e^{(z-y) \mathcal{A}} \mathcal{B C} \tilde{C}_{l}(x, z)=e^{-y \mathcal{A}} \mathcal{D} \omega(x), \\
\tilde{C}_{l}(x, y)-\int_{y}^{-2 x} d z e^{(y-z) \mathcal{A}} \mathcal{B C} C_{l}(x, z)=e^{(2 x+y) \mathcal{A}} \mathcal{D} \omega(x),
\end{gathered}
$$

implying (2.6), where $C_{l}\left(x, 0^{+}\right)=\tilde{C}_{l}\left(x,(-2 x)^{-}\right)=\mathcal{D} \omega(x)$. We thus get (2.9), where

$$
\begin{gathered}
{\left[\begin{array}{cc}
I & 0 \\
{\left[e^{2 x \mathcal{E}}\right]_{21}} & {\left[e^{2 x \mathcal{E}}\right]_{22}}
\end{array}\right]\left[\begin{array}{l}
h_{1} \\
h_{2}
\end{array}\right]=\left(\left[\begin{array}{ll}
I & 0 \\
0 & 0
\end{array}\right]+\left[\begin{array}{ll}
0 & 0 \\
0 & I
\end{array}\right] e^{2 x \mathcal{E}}\right)} \\
{\left[\begin{array}{l}
h_{1} \\
h_{2}
\end{array}\right]=\left[\begin{array}{c}
\mathcal{D} \omega(x) \\
\mathcal{D} \omega(x)
\end{array}\right] .}
\end{gathered}
$$

Now note that the matrix $\left[e^{2 x \mathcal{E}}\right]_{22}$ is nonsingular; otherwise the homogeneous counterpart of (2.12) would have a nontrivial solution, which would contradict Theorem A.1. In that case

$$
\left[\begin{array}{l}
h_{1} \\
h_{2}
\end{array}\right]=\left[\begin{array}{cc}
I & 0 \\
-\left[e^{2 x \mathcal{E}}\right]_{22}^{-1}\left[e^{2 x \mathcal{E}}\right]_{21} & {\left[e^{2 x \mathcal{E}}\right]_{22}^{-1}}
\end{array}\right]\left[\begin{array}{l}
I \\
I
\end{array}\right] \mathcal{D} \omega(x) .
$$

Consequently, $B_{l}\left(x, 0^{+}\right)=\mathcal{C} C_{l}\left(x, 0^{+}\right)=\mathcal{D} \omega(x)$, where

$$
\omega(x)=C_{l}\left(x,(-2 x)^{-}\right)=k+\mathcal{B}=\left[e^{2 x \mathcal{E}}\right]_{22}^{-1}\left(I-\left[e^{2 x \mathcal{E}}\right]_{21}\right) \mathcal{D} \omega(x)+\mathcal{B} .
$$

As a result, we obtain for $x<0$

$$
\begin{aligned}
B_{l}\left(x, 0^{+}\right)= & \mathcal{D} \omega(x)=U(x) \mathcal{B} \\
= & \left(I-\mathcal{D}\left[e^{2 x \mathcal{E}}\right]_{22}^{-1}\left(I-\left[e^{2 x \mathcal{E}}\right]_{21}\right)\right)^{-1} \mathcal{B} \\
Q(x)= & 4 U(x) \mathcal{D}\left[e^{2 x \mathcal{E}}\right]_{22}^{-1} \\
& \left\{\left[\mathcal{E} e^{2 x \mathcal{E}}\right]_{22}\left[e^{2 x \mathcal{E}}\right]_{22}^{-1}\left(I-\left[e^{2 x \mathcal{E}}\right]_{21}\right)+\left[\mathcal{E} e^{2 x \mathcal{E}}\right]_{21}\right\} U(x) \mathcal{B},
\end{aligned}
$$

where $U(x)=\left(I-\mathcal{D}\left[e^{2 x \mathcal{E}}\right]_{22}^{-1}\left(I-\left[e^{2 x \mathcal{E}}\right]_{21} 0\right)\right)^{-1}$ is nonsingular; otherwise a nonunique $\omega(x)$ may result. 
We now present two illustrative examples.

Example 2.1 Let $R(k)=i \xi /(k+i \eta)$ where $\eta>0$ and $0 \neq \xi \in[-\eta, \eta)$. Then $Q(x) \equiv 0$ for $x>0$. Moreover, $\mathcal{A}=[-\eta], \mathcal{B}=[\xi]$ and $\mathcal{C}=[1]$. Further,

$$
\mathcal{E}=\left[\begin{array}{cc}
-\eta & -\xi \\
\xi & \eta
\end{array}\right]
$$

so that $\mathcal{E}^{2}=\gamma^{2} I$ with $\gamma=\sqrt{\eta^{2}-\xi^{2}}$. Using the identity

$$
e^{y \mathcal{E}}= \begin{cases}\cosh (y \gamma) I+\frac{\sinh (y \gamma)}{\gamma} \mathcal{E}, & 0<|\xi|<\eta \\ I+y \mathcal{E}, & \xi=-\eta,\end{cases}
$$

we get for $0<|\xi|<\eta$

$$
e^{y \mathcal{E}}=\left[\begin{array}{cc}
\cosh (\gamma y)-\eta \frac{\sinh (\gamma y)}{\gamma} & -\xi \frac{\sinh (\gamma y)}{\gamma} \\
\xi \frac{\sinh (\gamma y)}{\gamma} & \cosh (\gamma y)+\eta \frac{\sinh (\gamma y)}{\gamma}
\end{array}\right]
$$

and for $\xi=-\eta, e^{y \mathcal{E}}=\left[\begin{array}{cc}1-\eta y & \eta y \\ -\eta y & 1+\eta y\end{array}\right]$. From (2.10) and using that $C_{l}(x, y)=B_{l}(x, y)$ when $x<0$, we get (2.9), where

$$
\left[\begin{array}{cc}
\cosh (2 \gamma x)-\eta \frac{\sinh (2 \gamma x)}{\gamma} & -\xi \frac{\sinh (2 \gamma x)}{\gamma} \\
0 & 1
\end{array}\right]\left[\begin{array}{l}
h_{1} \\
h_{2}
\end{array}\right]=\left[\begin{array}{c}
-\xi \\
-\xi
\end{array}\right],
$$

and therefore, as $B_{l}\left(x, 0^{+}\right)=h_{1}$, we get for $0<|\xi|<\eta$ and $x<0$

$$
\begin{aligned}
B_{l}\left(x, 0^{+}\right) & =\frac{-\xi[\gamma-\xi \sinh (-2 \gamma x)]}{\gamma \cosh (-2 \gamma x)+\eta \sinh (-2 \gamma x)}, \\
Q(x) & =4 \xi \gamma^{2} \frac{\xi+\eta \cosh (-2 \gamma x)+\gamma \sinh (-2 \gamma x)}{[\gamma \cosh (-2 \gamma x)+\eta \sinh (-2 \gamma x)]^{2}}-2 \xi \delta(x),
\end{aligned}
$$

where $\delta(x)$ is Dirac's delta function. For $\xi=-\eta$ we get $h_{1}=h_{2}=\eta$ and $B_{l}\left(x, 0^{+}\right)=\eta$, so that $Q(x)=2 \eta \delta(x)$.

One may also compute $B_{r}(x, y)$ from (1.4b), using the fact that (1.4b) has a separable kernel when $x<0$. As a result, we obtain

$$
B_{r}(x, y)=\frac{2 \xi \gamma e^{-\gamma(y-2 x)}}{\eta+\gamma-\xi e^{2 \gamma x}} .
$$

We find the same potential $Q(x)$, because $B_{l}(x, y)+B_{r}(x, y)=\eta-\gamma$ for $x<0$. 
Example 2.2 Let $R(k)=\xi \eta^{2} /\left(k^{2}+\eta^{2}\right)$ where $\eta>0$ and $0 \neq \xi \in[-1,1)$ and bound states are absent. Then

$$
\mathcal{A}=\left[\begin{array}{cc}
\eta & 0 \\
0 & -\eta
\end{array}\right], \quad \mathcal{B}=-\frac{\eta \xi}{2}\left[\begin{array}{c}
1 \\
-1
\end{array}\right], \quad \mathcal{C}=\left[\begin{array}{ll}
1 & 1
\end{array}\right], \quad \mathcal{D}=-\frac{\xi}{4}\left[\begin{array}{ll}
1 & 0 \\
0 & 0
\end{array}\right]
$$

while $\hat{R}(z)=(\xi \eta / 2) e^{-\eta|z|}$. For $x>0$ we easily solve (1.4a) and obtain

$$
B_{l}(x, y)=\frac{-2 \xi \eta e^{-(2 x+y) \eta}}{4+\eta e^{-2 x \eta}}, \quad Q(x)=\frac{-32 \xi \eta^{2} e^{-2 x \eta}}{\left[4+\xi e^{-2 x \eta}\right]^{2}}
$$

Letting $E_{24}$ be the matrix obtained from the $4 \times 4$ unit matrix by interchanging the second and fourth columns, we obtain

$$
E_{24} \mathcal{E} E_{24}=\eta T_{\xi}=\eta\left[\begin{array}{cccc}
1 & \xi / 2 & \xi / 2 & 0 \\
\xi / 2 & 1 & 0 & \xi / 2 \\
-\xi / 2 & 0 & -1 & -\xi / 2 \\
0 & -\xi / 2 & -\xi / 2 & -1
\end{array}\right]
$$

so that $E_{24} \mathcal{E}^{2} E_{24}=\eta^{2}\left[S_{\xi} \oplus S_{\xi}\right]$, where $S_{\xi}=\left[\begin{array}{ll}1 & \xi \\ \xi & 1\end{array}\right]$. Then

$$
E_{24} e^{y \mathcal{E}} E_{24}=\left[\cosh \left(y \eta S_{\xi}^{1 / 2}\right) \oplus \cosh \left(y \eta S_{\xi}^{1 / 2}\right)\right]
$$

$$
+\left[\sinh \left(y \eta S_{\xi}^{1 / 2}\right) \oplus \sinh \left(y \eta S_{\xi}^{1 / 2}\right)\right]\left[S_{\xi}^{-1 / 2} \oplus S_{\xi}^{-1 / 2}\right] T_{\xi},
$$

where for $a_{\xi}=\sqrt{1+\xi}, b_{\xi}=\sqrt{1-\xi}$ and $j=0,1,2, \ldots$

$$
S_{\xi}^{j / 2}=\frac{1}{2}\left[\begin{array}{cc}
a_{\xi}^{j}+b_{\xi}^{j} & a_{\xi}^{j}-b_{\xi}^{j} \\
a_{\xi}^{j}-b_{\xi}^{j} & a_{\xi}^{j}+b_{\xi}^{j}
\end{array}\right], \quad S_{\xi}^{-j / 2}=\frac{1}{2 a_{\xi}^{j} b_{\xi}^{j}}\left[\begin{array}{cc}
a_{\xi}^{j}+b_{\xi}^{j} & b_{\xi}^{j}-a_{\xi}^{j} \\
b_{\xi}^{j}-a_{\xi}^{j} & a_{\xi}^{j}+b_{\xi}^{j}
\end{array}\right]
$$

Applying the similarity $\frac{1}{\sqrt{2}}\left(\left[\begin{array}{rr}1 & 1 \\ -1 & 1\end{array}\right] \oplus\left[\begin{array}{rr}1 & 1 \\ -1 & 1\end{array}\right]\right)$ to (2.14), we find

$$
e^{y \mathcal{E}}=\frac{1}{2}\left[\begin{array}{rrrr}
T_{11}+T_{44} & T_{11}-T_{44} & T_{13}-T_{24} & T_{13}+T_{24} \\
T_{11}-T_{44} & T_{11}+T_{44} & T_{13}+T_{24} & T_{13}-T_{24} \\
-T_{13}+T_{24} & -T_{13}-T_{24} & T_{33}+T_{22} & T_{33}-T_{22} \\
-T_{13}-T_{24} & -T_{13}+T_{24} & T_{33}-T_{22} & T_{33}+T_{22}
\end{array}\right]
$$


where

$$
\begin{cases}T_{11}=\cosh \left(y \eta a_{\xi}\right)+\left(1+\frac{\xi}{2}\right) \frac{\sinh \left(y \eta a_{\xi}\right)}{a_{\xi}}, & T_{44}=\cosh \left(y \eta b_{\xi}\right)-\left(1-\frac{\xi}{2}\right) \frac{\sinh \left(y \eta b_{\xi}\right)}{b_{\xi}} \\ T_{22}=\cosh \left(y \eta b_{\xi}\right)+\left(1-\frac{\xi}{2}\right) \frac{\sinh \left(y \eta b_{\xi}\right)}{b_{\xi}}, & T_{33}=\cosh \left(y \eta a_{\xi}\right)-\left(1+\frac{\xi}{2}\right) \frac{\sinh \left(y \eta a_{\xi}\right)}{a_{\xi}} \\ T_{13}=\frac{\xi}{2} \frac{\sinh \left(y \eta a_{\xi}\right)}{a_{\xi}}, & T_{24}=\frac{\xi}{2} \frac{\sinh \left(y \eta b_{\xi}\right)}{b_{\xi}}\end{cases}
$$

and the $y$-dependence has not been written. Using $\mathcal{Q}_{\mathcal{A}}=\operatorname{diag}(1,0,0,1)$ and $I-\mathcal{Q}_{\mathcal{A}}=\operatorname{diag}(0,1,1,0)$, we obtain the invertibility of the matrix

$$
\mathcal{Q}_{\mathcal{A}}+\left(I-\mathcal{Q}_{\mathcal{A}}\right) e^{2 x \mathcal{E}}
$$

$$
=\left[\begin{array}{cccc}
1 & 0 & 0 & 0 \\
\frac{1}{2}\left(T_{11}-T_{44}\right) & \frac{1}{2}\left(T_{11}+T_{44}\right) & \frac{1}{2}\left(T_{13}+T_{24}\right) & \frac{1}{2}\left(T_{13}-T_{24}\right) \\
\frac{1}{2}\left(-T_{13}+T_{24}\right) & -\frac{1}{2}\left(T_{13}+T_{24}\right) & \frac{1}{2}\left(T_{33}+T_{22}\right) & \frac{1}{2}\left(T_{33}-T_{22}\right) \\
0 & 0 & 0 & 1
\end{array}\right],
$$

where $T_{i j}=T_{i j}(2 x)$, because the determinant of the $2 \times 2$ middle block

$$
\Delta(x, \eta, \xi)=\frac{1}{2}\left[\cosh \left(2 x \eta a_{\xi}\right) \cosh \left(2 x \eta b_{\xi}\right)+\frac{\sinh \left(2 x \eta a_{\xi}\right) \sinh \left(2 x \eta b_{\xi}\right)}{a_{\xi} b_{\xi}}\right]>0 .
$$

Writing $[S(x, \eta, \xi)]_{i, j=1}^{4}$ for the inverse of the matrix in (2.15), we obtain

$$
\begin{aligned}
& B_{l}\left(x, 0^{+}\right)=\left[\begin{array}{ll}
1 & 1
\end{array}\right] \\
& {\left[\begin{array}{ll}
S_{11}(x, \eta, \xi)+S_{13}(x, \eta, \xi) & S_{12}(x, \eta, \xi)+S_{14}(x, \eta, \xi) \\
S_{21}(x, \eta, \xi)+S_{23}(x, \eta, \xi) & S_{22}(x, \eta, \xi)+S_{24}(x, \eta, \xi)
\end{array}\right]\left[\begin{array}{l}
{[h(x)]_{1}} \\
{[h(x)]_{2}}
\end{array}\right],}
\end{aligned}
$$

where $h(x)=\mathcal{D} \omega(x)-P_{\mathcal{A}}^{(-)} \mathcal{B}$. Since $S_{11}=1$ and $S_{12}=S_{13}=S_{14}=0$, we get

$$
\begin{aligned}
B_{l}\left(x, 0^{+}\right)= & -\frac{\xi}{4}\left(1+S_{21}(x, \eta, \xi)+S_{23}(x, \eta, \xi)\right)[\omega(x)]_{1} \\
& -\frac{\eta \xi}{2}\left(S_{22}(x, \eta, \xi)+S_{24}(x, \eta, \xi)\right)
\end{aligned}
$$

where it suffices to compute the first component $[\omega(x)]_{1}$ of $\omega(x)$. Then $Q(x)$ will follow using (1.9) for $x<0$. Since $R(k)=o(1 / k)$ as $k \rightarrow \infty$, there will not be any delta function terms in the potential [15]. We omit the rather cumbersome computation of $[\omega(x)]_{1}$. 


\section{Solution of the Inverse Problem with Bound States}

\section{A. Preliminaries}

Let the (simple) poles of $T(k)$ occur at $i \kappa_{j}$. let the corresponding residues and bound state constants be $t_{j}$ and $c_{j}(j=1, \ldots, \mathcal{N})$, and let $\kappa_{1}>\cdots>\kappa_{\mathcal{N}}$. Considering $T(k)$ on the positive imaginary axis where $T(k) \rightarrow 1$ as $k \rightarrow$ $+i \infty, T(k)=\overline{T(-\bar{k})} \in \mathbf{R}$ and $-i t_{j}=\lim _{\kappa \rightarrow \kappa_{j}}\left(\kappa-\kappa_{j}\right) T(i \kappa)$, we see that $(-1)^{j-1}\left(-i t_{j}\right)>0$, since for every pole $i \kappa_{j}, \kappa_{j}$ is a sign change of $\kappa \mapsto 1 / T(i \kappa)$. Further, $f_{l}\left(i \kappa_{j}, x\right)=e^{-\kappa_{j} x}+o(1)$ as $x \rightarrow+\infty, f_{r}\left(i \kappa_{j} x\right)=e^{\kappa_{j} x}+o(1)$ as $x \rightarrow-\infty, c_{j}=f_{r}\left(i \kappa_{j}, x\right) / f_{l}\left(i \kappa_{j}, x\right)$ and the fact that $f_{l}\left(i \kappa_{j}, x\right)$ has $j-1$ simple zeros (which follows from the usual cscillation theorems [14]), imply that $(-1)^{j-1} c_{j}>0$. Consequently,

$$
d_{j}=-i t_{j} c_{j}>0, \quad j=1, \ldots, \mathcal{N} .
$$

\section{B. Marchenko Equation for Reflectionless Potentials}

When $\hat{R}(z) \equiv 0,(1.4 \mathrm{a})$ reduces to

$$
B_{l}(x, y)=-\sum_{j=1}^{\mathcal{N}} d_{j} e^{-(2 x+y) \kappa_{j}}\left[1+u_{j}(x)\right]
$$

where

$$
u_{j}(x)=\int_{0}^{\infty} d z e^{-\kappa_{j} z} B_{l}(x, z)
$$

$$
\sum_{j=1}^{\mathcal{N}}\left\{\delta_{i j}+\frac{d_{j} e^{-2 x \kappa_{j}}}{\kappa_{i}+\kappa_{j}}\right\} u_{j}(x)=-\sum_{j=1}^{\mathcal{N}} d_{j} \frac{e^{-2 x \kappa_{j}}}{\kappa_{i}+\kappa_{j}}, \quad i=1, \ldots, \mathcal{N} .
$$

We now easily see that $\left(d_{j} e^{-2 \kappa_{j}} /\left(\kappa_{i}+\kappa_{j}\right)\right)_{i, j=1}^{\mathcal{N}}$ is similar to the real symmetric matrix $M(x)$ with entries

$$
[M(x)]_{i j}=\frac{\sqrt{d_{i} d_{j}} e^{-\left(\kappa_{i}+\kappa_{j}\right) x}}{\kappa_{i}+\kappa_{j}} .
$$

This matrix is positive semidefinite, because for every $\xi=\left(\xi_{1}, \ldots, \xi_{\mathcal{N}}\right) \in \mathbf{C}^{\mathcal{N}}$

$$
(M(x) \xi, \xi)=\int_{0}^{\infty} d z\left|\sum_{i=1}^{\mathcal{N}} \xi_{i} \sqrt{d_{i}} e^{-\kappa_{i}(z+x)}\right|^{2} \geq 0
$$

Hence (3.2) is uniquely solvable and

$$
B_{l}(x, y)=\sum_{i, j=1}^{\mathcal{N}} \sqrt{d_{i} d_{j}} e^{-\kappa_{i} y} e^{-\left(\kappa_{i}+\kappa_{j}\right) x}\left[(I+M(x))^{-1}\right]_{i j} .
$$


Consequently,

$$
\begin{aligned}
Q(x)= & 2 \sum_{i, j=1}^{\mathcal{N}} \sqrt{d_{i} d_{j}} e^{-\left(\kappa_{i}+\kappa_{j}\right) x}+\left\{\left(\kappa_{i}+\kappa_{j}\right)\left[(I+M(x))^{-1}\right]_{i j}\right. \\
& \left.+\left[(I+M(x))^{-1}\left(\frac{d}{d x} M(x)\right)(I+M(x))^{-1}\right]_{i j}\right\} .
\end{aligned}
$$

\section{Formalism and Solution of the Marchenko Equation}

The integral kernel function $\hat{S}_{l}(z)$ can be written in the form (1.14), where $\breve{\mathcal{A}}, \breve{\mathcal{B}}$ and $\breve{\mathcal{C}}$ are given by (1.15), $\breve{\beta}=\left[\beta-d_{1} \ldots-d_{\mathcal{N}}\right]^{T}$ and $\breve{\gamma}=[\gamma 1 \ldots 1]^{T}$, and $d_{1}, \ldots, d_{\mathcal{N}}$ are positive constants [cf. (3.1)]. Then

$$
P_{\breve{\mathcal{A}}}^{(+)}=P_{\mathcal{A}}^{(+)} \oplus I_{\mathbf{C}^{\mathcal{N}}}, \quad P_{\breve{\mathcal{A}}}^{(-)}=P_{\mathcal{A}}^{(-)} \oplus 0_{\mathbf{C}^{\mathcal{N}}} .
$$

We define $\breve{\mathcal{D}}$ as in the statement of Theorem 1.2. With these definitions, one can repeat Subsections 2.c and 2.d when there are $\mathcal{N}$ bound states, where $\mathcal{A}, \mathcal{B}, \mathcal{C}, \mathcal{D}$, $\beta$ and $\gamma$ are to be replaced by $\breve{\mathcal{A}}, \breve{\mathcal{B}}, \breve{\mathcal{C}}, \breve{\mathcal{D}}, \breve{\beta}$ and $\breve{\gamma}$, respectively. As there is no conceptual problem, it will not be discussed at great length.

We limit ourselves to a few remarks. Instead of (1.8), we now have the Marchenko equation

$$
\begin{aligned}
& B_{l}(x, y)-\int_{0}^{\infty} d z \breve{\mathcal{C}} E(2 x+y+z ;-\breve{\mathcal{A}}) \breve{\mathcal{B}} B_{l}(x, z) \\
& =\breve{\mathcal{C}} E(2 x+y ;-\breve{\mathcal{A}}) \breve{\mathcal{B}}, \quad y>0,
\end{aligned}
$$

where the potential $Q(x)$ follows from (1.9). When $x>0,(3.3)$ has a separable kernel and its solution $B_{l}(x, y)$ and the corresponding potential $Q(x)$ are given in the statement of Theorem 1.2. On the other hand, when $x<0$, the solution of (3.3) for $y=0^{+}$is given by (2.7), where $\mathcal{A}, \mathcal{B}, \mathcal{C}$, and $\mathcal{D}$ are to be replaced by $\breve{\mathcal{A}}, \breve{\mathcal{B}}$, $\breve{\mathcal{C}}$, and $\breve{\mathcal{D}}$, respectively; these replacements are also to be made in the expressions for $\mathcal{E}$ [thus converting (2.1) into (3.4) below], $\mathcal{Q}_{\mathcal{A}}$ and $\omega(x)$. The potential $Q(x)$ follows by applying (1.9). When $x<0$ and $R(k)$ is analytic in $\mathbf{C}^{-}$, the solution of (3.3) for $y=0^{+}$and the potential $Q(x)$ are given by

$$
\begin{aligned}
B_{l}\left(x, 0^{+}\right)= & \left(I-\breve{\mathcal{D}}\left[e^{2 x \breve{\mathcal{E}}}\right]_{22}^{-1}\left(I-\left[e^{2 x \breve{\mathcal{E}}}\right]_{21}\right)\right)^{-1} \breve{\mathcal{B}} ; \\
Q(x)= & 4 \breve{U}(x) \breve{\mathcal{D}}\left[e^{2 x \breve{\mathcal{E}}}\right]_{22}^{-1} \\
& \left\{\left[\breve{\mathcal{E}} e^{2 x \breve{\mathcal{E}}}\right]_{22}\left[e^{2 x} \breve{\mathcal{E}}\right]_{22}^{-1}\left(I-\left[e^{2 x \breve{\mathcal{E}}}\right]_{21}\right)+\left[\breve{\mathcal{E}} e^{2 x} \breve{\mathcal{E}}\right]_{21}\right\} \breve{U}(x) \breve{\mathcal{B}},
\end{aligned}
$$

where $\breve{U}(x)=\left(I-\breve{\mathcal{D}}\left[e^{2 x \breve{\mathcal{E}}}\right]_{22}^{-1}\left(I-\left[e^{2 x \breve{\mathcal{E}}}\right]_{21}\right)\right)^{-1}$ is nonsingular. Here

$$
\breve{\mathcal{E}}=\left[\begin{array}{cc}
\breve{\mathcal{A}} & -\breve{\mathcal{B}} \breve{\mathcal{C}} \\
\breve{\mathcal{B}} \breve{\mathcal{C}} & -\breve{\mathcal{A}}
\end{array}\right] .
$$


When $x<0$ and $R(k)$ is analytic in $\mathbf{C}^{+}$, one does not find an expression analogous to (2.11), because $\breve{\mathcal{A}}$ has eigenvalues in bothı the left and the right half-plane.

\section{Appendix A. Well-posedness of the Marchenko Equations}

The main result of the Appendix is well-known [16, 18]. We give a short proof. For real $\hat{\rho} \in L^{1}\left(\mathbf{R}^{+}\right)$, define

$$
\left(\mathcal{L}_{\hat{\rho}} f\right)(y)=\int_{0}^{\infty} d z \hat{\rho}(y+z) f(z) .
$$

Then $\mathcal{L}_{\hat{\rho}}$ is a compact operator on each of the Banach spaces $L^{p}\left(\mathbf{R}^{+}\right)(1 \leq$ $p \leq+\infty)$ and on $B C([0,+\infty))$, the space of bounded continuous complexvalued functions on $[0,+\infty)$ with supremum norm. Moreover, $\mathcal{L}_{\hat{\rho}}$ maps $L^{\infty}\left(\mathbf{R}^{+}\right)$ into $B C([0,+\infty))$. Let $H_{ \pm}^{p}(\mathbf{R})$ be the closed subspaces of $L^{p}(\mathbf{R})$ consisting of those $L^{p}$-functions that have an analytic continuation to $\mathbf{C}^{ \pm}$(cf. [25]). Then $H_{+}^{p}(\mathbf{R}) \oplus H_{-}^{p}(\mathbf{R})=L^{p}(\mathbf{R})(1<p<+\infty)$, where the direct sum is orthogonal when $p=2$. Letting $\tau_{ \pm}^{(H)}: H_{ \pm}^{2}(\mathbf{R}) \rightarrow L^{2}(\mathbb{R})$ denote the natural embeddings and $\pi_{ \pm}^{(H)}: L^{2}(\mathbf{R}) \rightarrow H_{ \pm}^{2}(\mathbf{R})$ the orthogonal projections, $(\mathcal{F} h)(k)=\int_{-\infty}^{\infty} d t e^{i k t} h(t)$ the Fourier transform, and $(J h)(k)=h(-k)$ the sign inversion, we have on $L^{2}\left(\mathbf{R}^{+}\right)$

$$
\mathcal{L}_{\hat{\rho}}=\mathcal{F}^{-1} \pi_{+}^{(H)} J M_{\rho} \tau_{+}^{(H)} \mathcal{F},
$$

where $\left(M_{\rho} h\right)(k)=\rho(k) h(k)$ is the operator of multiplication by $\rho(k)=\int_{-\infty}^{\infty}$ $d t e^{-i k t} \hat{\rho}(t)$. By Nehari's theorem [30, 31],

$$
\left\|\mathcal{L}_{\hat{\rho}}\right\|_{L^{2}\left(\mathbf{R}^{+}\right)}=\inf _{u \in H_{+}^{\infty}(\mathbf{R})}\|\rho-u\|_{\infty}
$$

The following result implies the unique solvability of the Marchenko equations (1.4a) and (1.4b) on a variety of function spaces.

Theorem A.1 On the Banach spaces $B C([0,+\infty))$ and $L^{p}\left(\mathbf{R}^{+}\right)(1 \leq p \leq$ $+\infty)$, the integral operators with real synmetric kernels $\hat{S}_{l}(2 x+y+z)$ and $\hat{S}_{r}(-2 x+y+z)$ have their eigenvalues in the interval $(-1,+\infty)$.

Proof: Without bound states, $|R(k)|<1$ for $0 \leq k \in \mathbf{R}$ and $R(0) \in[-1,1)$ imply that $|R(k)+\varepsilon|<\mathrm{l}$ for $k \in \mathbf{R}$ for some suitable $\varepsilon \geq 0$ not depending on $k$. Then (A.1) implies that $\mathcal{L}_{\hat{\rho}}$ is a strict contraction on $L^{2}\left(\mathbf{R}^{+}\right)$. Since $d_{1}, \ldots, d_{\mathcal{N}}>0$, the integral kernel with kernel $\sum_{j=1}^{\mathcal{N}} d_{j} e^{-\kappa_{j}(2 x+y+z)}$ is positive selfadjoint on $L^{2}\left(\mathbf{R}^{+}\right)$. Hence, when bound states are taken into account, $\mathcal{L}_{\hat{S}_{l}}$ has its eigenvalues in $(-1,+\infty)$. The proof for $\mathcal{L}_{\hat{S}_{r}}$ is similar.

A simple Fredholm argument yields the same results in any of the other function spaces mentioned above. 
Corollary A.2 Let $c>0$. On the Banach spaces $L^{p}(0, c)(1 \leq p \leq+\infty)$ and $B C([0, c])$, the integral operators with real symmetric kernels $\hat{S}_{l}(2 x+y+z)$ and $\hat{S}_{r}(-2 x+y+z)$ have their eigenvalues in the interval $(-1,+\infty)$.

Proof: Let $\tau_{c}: L^{2}(0, c) \rightarrow L^{2}\left(\mathbf{R}^{+}\right)$denote the natural embedding and $\pi_{c}$ : $L^{2}\left(\mathbf{R}^{+}\right) \rightarrow L^{2}(0, c)$ the orthogonal projection. Then these integral operators can be written as $\pi_{c} \mathcal{L}_{\hat{S}_{l}} \tau_{c}$ and $\pi_{c} \mathcal{L}_{\hat{S}_{r}} \tau_{c}$, respectively, and hence when adding the identity one gets positive selfadjoint operators.

A simple Fredholm argument again yields the same results in any of the other Banach spaces of functions on $(0, c)$.

\section{References}

1. Z.S. Agranovich and V.A. Marchenko, The Inverse Problem of Scattering Theory. Kharkov Univ. Press, Kharkov, 1960 [English Translation: Gordon \& Breach, New York, 1963]. MR 28, \#5696.

2. T. Aktosun, M. Klaus and C. van der Mee, Explicit Wiener-Hopf factorization for certain non-rational matrix functions. Integral Equations and Operator Theory 15 (1992), 879-900. MR 93j:47030.

3. D. Alpay and I. Gohberg, Inverse spectral problems for differential operators with rational scattering matrix functions. J. Diff. Eqs. 118 (1995), 1-19. MR 96f:34121.

4. D. Alpay and I. Gohberg, Inverse scattering problem for differential operators with rational scattering matrix functions. In: Böttcher, A. and Gohberg, I. (Eds.), Singular Integral Operators and Related Topics. Joint German-Israeli Workshop, Tel Aviv, March 1-10, 1995. Birkhäuser OT 90, Basel, 1996; pp. 1-18. MR 97j:34112.

5. D. Alpay and I. Gohberg, Potentials associated to rational weights. In: Gohberg, I. and Lyubich, Yu. (Eds.), New Results in Operator Theory and its Applications. The Israel M. Glazman Memorial Volume, Birkhäuser OT 98, Basel, 1997; pp. 23-40. MR 99a:34218.

6. D. Alpay and I. Gohberg, State space method for inverse spectral problems. Progress in Systems and Control Theory 22 (1997), 1-16 MR 98d:34113.

7. D. Alpay, I. Gohberg and L. Sakhnovich, Inverse scattering problem for continuous transmission lines with rational reflection coefficient function. In: Gohberg, I., Lancaster, P. and Shivakumar, P. N. (Eds.), Recent Developments in Operator Theory and its Applications. International Conference in Winnipeg, October 2-6, 1994. Birkhäuser OT 87, Basel, 1996. MR 97f:340721.

8. D. Alpay and I. Gohberg, Inverse problem for Sturm-Liouville operators with rational reflection coefficient. Integral Equations and Operator Theory 30 (1998), 317-325. MR 1608685.

9. H. Bart, I. Gohberg and M.A. Kaashoek, Minimal Factorization of Matrix and Operator Functions. Birkhäuser OT 1, Basel, 1979. MR 81a:47001.

10. H. Bart, I. Gohberg and M.A. Kaashoek, Exponentially dichotomous operators and inverse Fourier transforms. Report 8511/M, Econometric Institute, Erasmus University of Rotterdam, The Netherlands, 1985.

11. H. Bart, I. Gohberg and M.A. Kaashoek, Wiener-Hopf factorization, inverse Fourier transforms and exponentially dichotomous operators. J. Funct. Anal. 68 (1986), 1-42. MR 88d:47052. 
12. R. Bhatia, Matrix Analysis. Graduate Texts in Mathematics 169, Springer, New York, 1997. MR 98i: 15003.

13. K. Chadan and P. Sabatier, Inverse Problems in Quantum Scattering Theory. Texts and Monographs in Physics, 2nd ed., Springer, New York, 1989. MR 90b:81002.

14. E.A. Coddington, and N. Levinson, Theory of Ordinary Differential Equations. McGraw-Hill, New York, 1955. MR 16, \#1022b.

15. P. Deift and E. Trubowitz, Inverse scatterin; ${ }^{3}$ on the line. Comm. Pure Appl. Math. 32 (1979), 121-251. MR 80e:34011.

16. W. Eckhaus and A. van Harten, The Inverse Scattering Transformation and the Theory of Solitons. North-Holland, Math. Studies 50 (1981). MR 83c:35101.

17. L.D. Faddeev, The inverse problem in the quantum theory of scattering. Uspekhi Matem. Nauk 14 (1959), 57-119 [English translation (in collaboration with B. Seckler): J. Math. Phys. 4 (1963), 72-104]. MR 22, \#1344 (Uspekhi); MR 26, \#7328 (JMP).

18. L.D. Faddeev, Properties of the $S$-matrix of the one-dimensional Schrödinger equation. Amer. Math. Soc. Transl. 2 (1964), 139-156 [Trudy Mat. Inst. Steklova 73 (1964), 314-336 (Russian)]. MR 31, \#2446.

19. I.M. Gel'fand and B.M. Levitan, On the determination of a differential equation from its spectral function. Dokl. Akad. Nauk SSSR 77(4) (1951), 557-560 [Russian]. MR 13, $240 \mathrm{f}$.

20. I.M. Gel'fand and B.M. Levitan, On the determination of a differential equation from its spectral function. Izv. Akad. Nauk SSSR 15 (1951), 309-360 [Russian]. MR 13, $558 \mathrm{f}$.

21. I. Gohberg, S. Goldberg and M.A. Kaashoek, Classes of Linear Operators. vol. I. Birkhäuser OT 49, Basel, 1990. MR 93d:47002.

22. I. Gohberg, M.A. Kaashoek and A.L. Salkhnovich, Canonical systems with rational spectral densities: Explicit formulas and applications. Math. Nachr. 194 (1998), 93-125. MR 1653082.

23. I. Gohberg, M.A. Kaashoek and A.L. Sakhnovich, Pseudocanonical systems with rational Weyl functions: Explicit formulas and applications. J. Diff. Eqs. 146 (1998), 375-398. MR 1631291.

24. I. Gohberg, M.A. Kaashoek and A.L. Sakhnovich, Sturm-Liouville systems with rational Weyl functions: Explicit formulas and applications. Integral Equations and Operator Theory 30 (1998), 338-377. MR 1608648.

25. K. Hoffman, Banach Spaces of Analytic Functions. Prentice-Hall, Englewood Cliffs, 1962; also: Dover Publ., New York, 1988. MR 24, \#A2844.

26. M. Klaus, Low-energy behaviour of the scatlering matrix for the Schrödinger equation on the line. Inverse Problems 4 (1988), 505-512. MR 89k:81185.

27. M.G. Krein, On the determination of a potential from its $S$-function. Dokl. Akad. Nauk SSSR 105(3) (1955), 433-436 [Russian]. NIR 17, $1210 \mathrm{~b}$.

28. V.A. Marchenko, Recovery of the potential energy from the phases of the scattered waves. Dokl. Akad. Nauk SSSR 104(5) (1955), 695-698 [Russian]. MR 17, 740d.

29. A. Melin, Operator methods for inverse scattering on the real line. Comm. in Partial Differential Equations 10(7) (1985), 677-766. MR 86f:35177.

30. J.R. Partington, An Introduction to Hankel Operators. London Math. Soc. Student Texts, vol. 13, Cambridge Univ. Press, Cambridge, 1988. MR 90c:47047.

31. S.C. Power, Hankel Operators on Hilbert Space. Research Notes in Mathematics 64, Pitman, Boston, 1982. MR 84e:47037. 
32. M. Rosenblum, On the operator equation $B X-X A=Q$, Duke Math. J. 23 (1956), 263-269. MR 18, 54d.

33. W.E. Roth, The equations $A X-Y B=C$ and $A X-X B=C$ in matrices. Proc. Amer. Math. Soc. 3 (1952), 392-396. MR 13, 900c.

34. M. Wadati, Generalized matrix form of the inverse scattering method. In Bullough, R.K. and Caudry, P. J. (Eds.), Solitons, Topics in current physics, vol. 17, pp. 287-299, Springer, Berlin, 1980.

35. M. Wadati and T. Kamijo, On the extension of inverse scattering method. Progress of Theor. Phys. 52 (1974), 397-414. MR 53, \#9964.

\author{
Cornelis van der Mee \\ Dipartimento di Matematica \\ Università di Cagliari \\ Via Ospedale 72 \\ 09124 Cagliari \\ Italy \\ corneliskrein.unica.it \\ http://krein.unica.it/ cornelis
}

MSC Primary 34A55, 81U40 